This is a postprint version of the following published document: Javier Mata; Ignacio de Miguel; Ramón J. Durán; Noemí Merayo; Sandeep Kumar Singh; Admela Jukan; Mohit Chamania. Application of Artificial Intelligence Techniques in Optical Networks. In: 2018 IEEE Photonics Society Summer Topical Meeting Series (SUM). DOI: https://doi.org/10.1109/PHOSST.2018.8456691

(C) 2018 IEEE. Personal use of this material is permitted. Permission from IEEE must be obtained for all other uses, in any current or future media, including reprinting/republishing this material for advertising or promotional purposes, creating new collective works, for resale or redistribution to servers or lists, or reuse of any copyrighted component of this work in other works. 


\section{Application of Artificial Intelligence Techniques in Optical Networks}

\author{
Javier Mata \\ Universidad de Valladolid \\ Valladolid, Spain \\ javier.mata@ ribera.tel.uva.es
}

\author{
Ignacio de Miguel \\ Universidad de Valladolid \\ Valladolid, Spain \\ ignacio.miguel@tel.uva.es
}

\author{
Ramón J. Durán \\ Universidad de Valladolid \\ Valladolid, Spain \\ rduran@tel.uva.es
}

\author{
Noemí Merayo \\ Universidad de Valladolid \\ Valladolid, Spain \\ noemer@tel.uva.es
}

\author{
Sandeep Kumar Singh \\ Technische Universität Carolo-Wilhelmina \\ zu Braunschweig \\ Braunschweig, Germany \\ sandeep.singh@tu-bs.de
}

\author{
Admela Jukan \\ Technische Universität Carolo-Wilhelmina \\ zu Braunschweig \\ Braunschweig, Germany \\ a.jukan@tu-bs.de
}

\author{
Mohit Chamania \\ ADVA Optical Networking \\ Berlin, Germany \\ MChamania@advaoptical.com
}

\begin{abstract}
We provide an overview of artificial intelligence techniques and their use in optical communication systems and networks with the aim of improving performance. Areas of application include optical transmission, performance monitoring, quality of transmission monitoring, as well as optical network planning and operation.
\end{abstract}

Keywords-artificial intelligence, machine learning, optical communications, optical networks

\section{INTRODUCTION}

Artificial intelligence (AI), as a scientific discipline, encompasses very diverse areas including search algorithms and optimization theory, local search algorithms and metaheuristics, game theory, knowledge-based reasoning and planning methods, statistical models and decision-making algorithms, as well as learning methods, most notably, machine learning [1]. Many of these techniques have been used in the optical networking arena for a long time, but their popularity, especially machine learning, is gaining momentum [2, 3]. Areas of application include optical transmission, performance monitoring, quality of transmission monitoring, as well as optical network planning and operation, to name a few.

The use of AI in optical networks provides indeed ample opportunities for automating operations and introducing intelligent decision making in network control and management, including issues like connection establishment, self-configuration and self-optimization, through prediction and estimation by utilizing present network state and historical data. AI can also help improve the configuration and operation of network devices, optical performance monitoring, modulation format recognition, fiber nonlinearities mitigation and quality of transmission (QoT) estimation, resulting in overall better network performance. Using AI in optical networks makes the networks cognitive [4]. Cognitive optical

Work partially supported by the Spanish Ministry of Economy and Competitiveness (TEC2014-53071-C3-2-P, TEC2017-84423-C3-1-P, and TEC2015-71932-REDT). networks are supported by an intelligent "brain" (or, a cognitive decision system, CDS) or intelligent controller functions implemented in Software Defined Networking (SDN) framework. The intelligent cognitive controller exploits the data collected from network monitors and additional data sources with the use of AI techniques to improve network performance. At the same time, AI also presents opportunities and challenges in automating operations and decision making due to large sample of collected data through heterogeneous sensors and network devices, and time-varying parameters, which we describe in subsequent sections while presenting its applications.

\section{Use CASES OF AI IN OPTICAL NETWORKING}

We have recently presented a comprehensive survey of the application of AI in optical networks, covering all AI-based methods from search algorithms to machine learning [2]. Fig. 1 summarizes the applications reviewed in [2]. A subsequent survey in this area, by Musumeci et al. [3], focuses on machine learning in optical networks specifically. Both surveys point to a broad range of use cases and services aided with AI-based methods.

Consider the already mentioned cognitive optical network based on SDN. As current networks are facing increased levels of heterogeneity in terms of services, devices and transmission technologies, the optimization of adjustable parameters and operational decisions presents a significant challenge. A cognitive SDN controller can have access to data collected by network sensors (such as traffic load, performance monitoring, quality of transmission of the connections and energy consumption), but also to business related data, like service level agreements (SLA), user profiles, capital and operation expenses, gains per service, and additional key performance indicators (KPIs). Cognitive optical networks are, thus, the next step in the evolution of intelligent optical networks by exploiting that information as described next. 


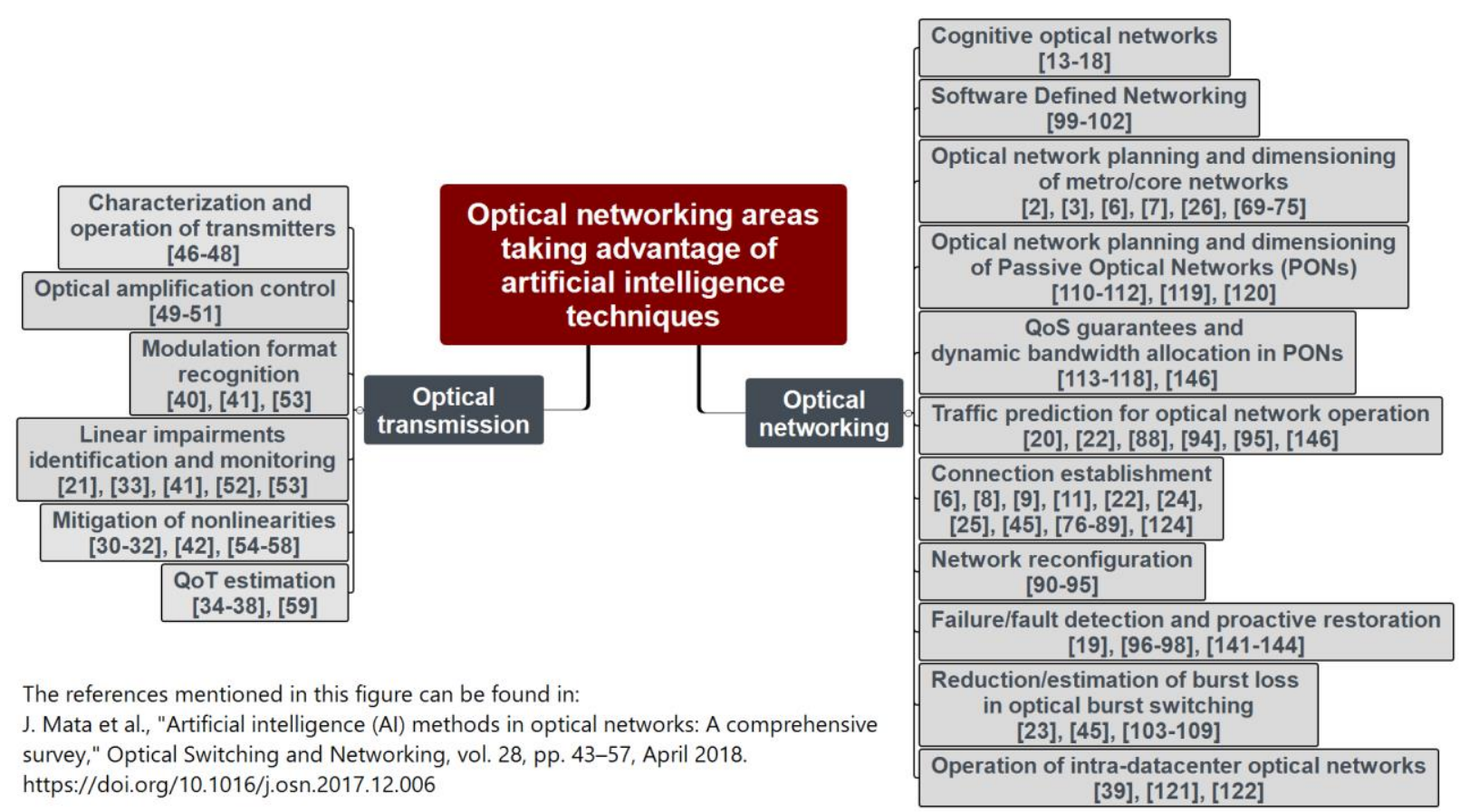

Fig. 1. Applications in optical networking taking advantage of AI techniques. The references shown in the figure correspond to those presented in [2].

The area of optical network planning and dimensioning has widely relied on the use of search algorithms and optimization theory, usually complemented by or extended with local search algorithms and metaheuristics, such as simulated annealing, swarm optimization and genetic algorithms. This is another important AI-enabled area. Here, all information collected during network operation can be exploited in the green field planning scenario as well as for network engineering purposes.

Individual optical devices can also benefit from AI-based concepts. For instance, AI can help in the autonomous adjustment of the operating point of amplifiers when dealing with dynamic variation of optical connections in the network, and receivers can employ AI for modulation format recognition. Also, machine learning can be incorporated into digital signal processing to mitigate nonlinearities in a more efficient way, allowing for more accurate symbol detection. A known challenge in network control and management, to adapt to the time-varying link performance parameters, can also be addressed with AI. This can help mitigating various effects, such as optical signal to noise ratio (OSNR), nonlinearity factors, chromatic dispersion (CD) and polarization mode dispersion (PMD). To this end, a number of machine learning methods (e.g., using neural networks) have already been proposed to estimate these parameters from signals received.

Finally, AI can be applied at the network operation level. On the one hand, traffic prediction models, e.g., based on time series analysis like the ARIMA (autoregressive integrated moving average) methods, or neural networks, can be used to plan dynamic establishment, hold-on and release of optical connections, as well as to trigger virtual topology reconfiguration procedures. The methods for path computation, resource allocation, and virtual topology reconfiguration orchestration processes, can rely on metaheuristics like simulated annealing and evolutionary methods like genetic algorithms or particle swarm optimization. On the other hand, it is worthy to note that it is important to ensure that connections to be established comply with QoT requirements and that the existing ones will continue complying with their own requirements if a new one is established. For that aim, a QoT estimation module, capable of providing fast and accurate predictions is a key element. Machine learning techniques like case-based reasoning, support vector machines, neural networks and random forests have proved their efficiency for this task. On the other hand, Bayesian inference or anomaly detection techniques have been used for failure detection and prevention, allowing for proactive actions.

In summary, artificial intelligence provides a number of opportunities in the area of optical communication and networking, and we expect that it will continue playing a crucial role in supporting technologies like space division multiplexing using multimode/multicore fibers as well as in the automation of network management operations.

\section{REFERENCES}

[1] S. Russell, P. Norvig, Artificial Intelligence: A Modern Approach, $3^{\text {rd }}$ Edition, Prentice Hall Press, 2009.

[2] J. Mata, I. de Miguel, R. J. Durán, N. Merayo, S. K. Singh, A. Jukan, and M. Chamania, "Artificial intelligence (AI) methods in optical networks: A comprehensive survey," Optical Switching and Networking, vol. 28, pp. 43-57, April 2018. https://doi.org/10.1016/j.osn.2017.12.006

[3] F. Musumeci, C. Rottondi, A. Nag, I. Macaluso, D. Zibar, M. Ruffini, and M. Tornatore, "A survey on application of machine learning techniques in optical networks," https://arxiv.org/abs/1803.07976.

[4] I. de Miguel et al., "Cognitive dynamic optical networks [Invited]," J. Opt. Commun. Netw., vol. 5, no. 10, pp. A107-A118, Oct. 2013. 